\title{
Idiopathic syringomyelia
}

INSERM

\section{Source}

INSERM. (1999). Orphanet: an online rare disease and orphan drug data base. Idiopathic syringomyelia. ORPHA:99858

Idiopathic syring omyelia is a rare, non-syndromic central nervous system malformation characterized by a long itudinally oriented fluid-filled cavity inside the spinal cord parenchyma or the central canal, without any readily identifiable cause. It is usually associated with pain, sensory and/or musculoskeletal disturbances, but it can also be an incidental and asymptomatic finding. 